\title{
Delayed presentation of bilateral diaphragmatic palsy following trauma
}

\author{
Aditi Joshi, Ketaki Utpat, Unnati Desai* and Ramesh N Bharmal \\ Associate professor and Incharge, Department of Pulmonary medicine, TNMC and BYL Nair Hospital, Mumbai, India
}

\begin{abstract}
The diaphragm is the main respiratory muscle, its dysfunction can lead to serious consequences. Diaphragm paralysis can be a result of various causes, most common of being injury to phrenic nerve following thoracic surgeries. Presentation can vary from a totally asymptomatic patient to disabling condition requiring mechanical ventilation. Clinically paradoxical breathing is evident. Spirometry shows drop in lung capacity, with significant decline in supine position, which is typical of diaphragmatic palsy. Treatment depends on the cause. Surgical approach of repair of diaphragm or nonsurgical approach of non-invasive ventilation has been successful. We report a case of 33-year-old male with bilateral diaphragm palsy, managed with non-invasive ventilation.
\end{abstract}

\section{Introduction}

The diaphragm is the primary respiratory muscle. Diaphragmatic dysfunction can be associated with respiratory symptoms, exercise intolerance, sleep disturbances, and in severe cases having negative impact on survival. The diagnosis and management of Unilateral and bilateral diaphragmatic dysfunction can be sometimes problematic due to its subtle presentation, relative rarity and difficulty in obtaining a physician confirmed diagnosis [1]. Symptoms often occur shortly after injury, may be delayed for many years, hence require a prolonged follow up. Unilateral diaphragm paralysis is often first suspected after the finding of an abnormally elevated hemidiaphragm on a chest radiograph, which can be defined as a right hemidiaphragm sitting $>2$ $\mathrm{cm}$ higher than its left counterpart or a left hemidiaphragm sitting equal or higher than the right hemidiaphragm. Bilateral diaphragm palsy is a rare cause of acute respiratory failure and is often related to phrenic nerve injury as well as varieties of neuropathy and myopathies, however the cause goes unidentified in two third of cases [2]. We herein report a case of post traumatic bilateral diaphragmatic paralysis.

\section{Case report}

A 33-year-old male with no known comorbidities presented to the emergency department of our hospital, with history of fall from a height of nearly 10-12 feet, followed by severe lower back pain, left ankle pain and swelling, inability to walk, bilateral lower limb numbness and tingling sensation. no h/o unconsciousness, convulsions, vomiting. There was no significant past history. On general examination patient was afebrile, there was no evidence of bleeding. Left ankle swelling and tenderness was present. Patient's vitals were stable. Respiratory system examination was unremarkable on presentation to the emergency department. Higher functions were normal, patient was conscious and oriented. Other routine blood investigations were within normal limits. Chest $\mathrm{x}$-ray was within normal limits. In view of severe back pain MRI of lumbosacral spine was done which was suggestive of compression fracture of the D 12 vertebral body with retropulsion of fractured fragments noted posteriorly into the spinal canal with narrowing and compression of spinal cord. Mild desiccation of L5-S1 disc was also noted. Patient was initially managed symptomatically under orthopedic care. Fifteen days post trauma, the patient complained of dyspnea, inability to lie flat owing to severe breathlessness, relieved in propped up or in sitting position. Patient was evaluated by respiratory team. On evaluation, patient was tachypneic with pulse rate of 110 per minute. Respiratory system examination revealed bilateral diminished breath sounds in lung bases. In the upright position, respiration was normal. In supine position, patient immediately complained of severe respiratory discomfort, and exhibited thoracoabdominal paradoxical breathing and the use of accessory muscles of respiration. No other abnormality was reported. Chest radiograph demonstrated elevation of hemidiaphragm, with bibasilar atelectasis, which was not evident on initial presentation (Figure 1). Arterial blood gas was done in sitting position, the values obtained were, $\mathrm{pH}$ of $7.35, \mathrm{PCO} 2$ of $46 \mathrm{mmHg}, \mathrm{Po} 2$ of $60 \mathrm{mmHg}, \mathrm{HCO} 3$ of $25 \mathrm{meq} / \mathrm{l}$. CT Chest was suggestive of basilar atelectasis with reduced lung volumes, with no other abnormality (Figure 2). There was no paradoxical motion on sniff test. Spirometry was performed in sitting and supine position. In sitting position FEV1/ FVC ratio was $106 \%$, FVC of $1.57 \mathrm{~L}$ (36 \% of predicted) and FEV1 of $1.37 \mathrm{~L}$ ( $37 \%$ of predicted), suggestive of restrictive abnormality. In supine position there was significant fall in lung volumes, with fall in FVC by $1.21 \mathrm{~L}$ with FVC of $0.36 \mathrm{~L}$ ( $8 \%$ of predicted) and FEV1 of $0.32 \mathrm{~L}$ ( $9 \%$ of predicted) (Figure $3 \mathrm{a}$ and $3 \mathrm{~b}$ ). MRI cervical spine showed block vertebrae at C2 - C3 and C5 - C6 level with fusion posterior elements, which was believed to be clinically non-significant. The clinical, radiological and spirometry findings demonstrated the diagnosis of bilateral diaphragm palsy secondary to his prior fall. During his stay in hospital patient was managed with Bipap overnight and during daytime. He gradually improved symptomatically and was discharged with home Bipap.

${ }^{\star}$ Correspondence to: Unnati Desai, Associate professor and Incharge, Department of Pulmonary medicine, TNMC and BYL Nair Hospital, Mumbai, India, E-mail: unnati_desai82@yahoo.co.in

Key words: diaphragmatic palsy, respiratory failure

Received: February 08, 2021; Accepted: February 18, 2021; Published: February 24,2021 


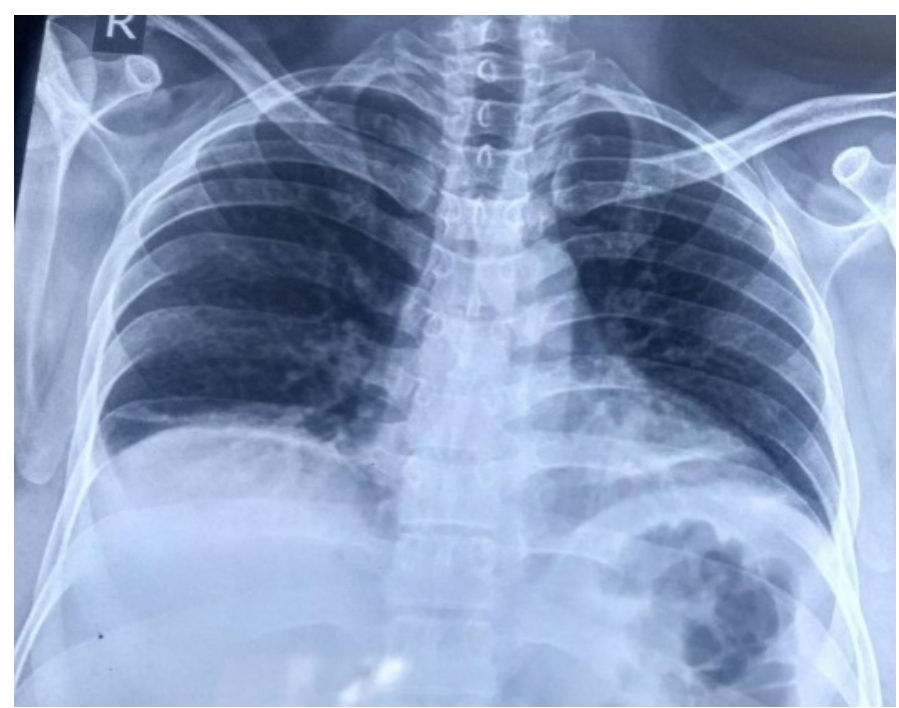

Figure 1. Chest x-ray showing bilateral elevated haemidiphragm - with reduced lung volume

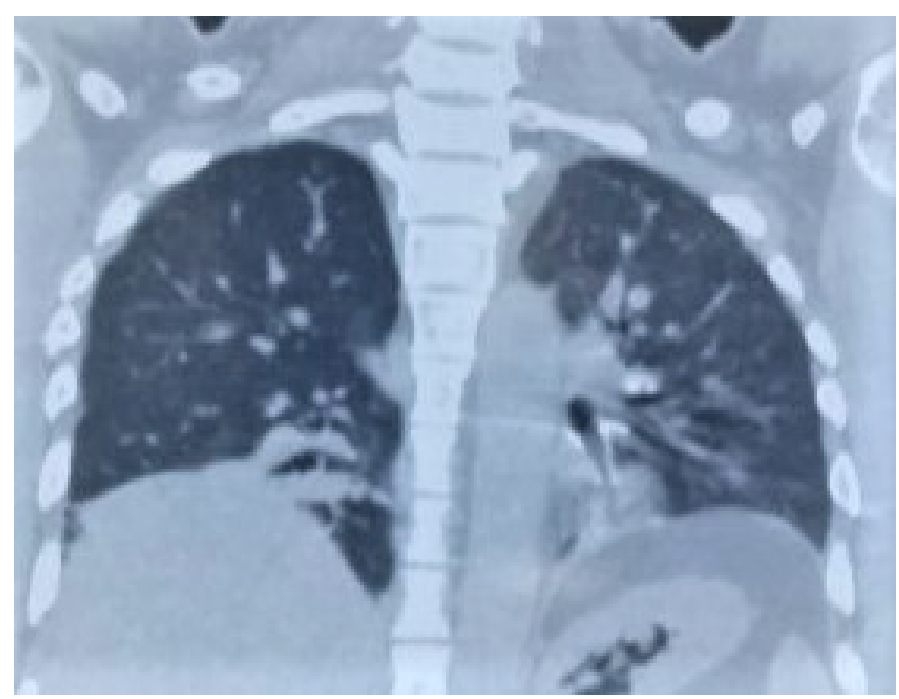

Figure 2. CECT - chest showing elevated hemidiphragms, with basilar atelectasis and reduced lung volumes



(A)

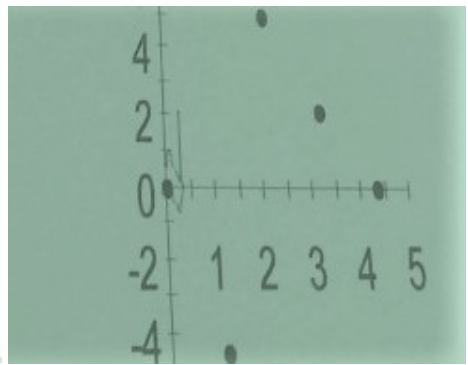

(B)
Figure 3. (A) Flow volume loop in sitting position, suggesting restriction (B) Flow volume loop in supine position showing drastic fall in lung volume with decline in FVC by 1.21 litre

\section{Discussion}

Though the diaphragm forms one continuous musculotendinous sheet between the thorax and the abdomen, both the diaphragms work as an independent unit, paralysis of one phrenic nerve might be expected to have little effect because tension in the whole diaphragm should be maintained by activity in the contralateral phrenic nerve. When bilateral diaphragm palsy develops, it can lead to severe morbidity. Thoracic surgery, or intrathoracic malignancy are the most common cause of unilateral or bilateral diaphragmatic palsy, but can also result from trauma, and various inflammatory disorder such as pneumonia or herpes zoster infection. Diaphragm paralysis may also result from myopathies, such as dermatomyositis, limb girdle muscular dystrophy, mixed connective tissue disease, acid maltase deficiency, malnutrition, or amyloidosis. Neuropathic diseases such as poliomyelitis, multiple sclerosis, amyotrophic lateral sclerosis, and Guillain-Barre' syndrome may also lead to diaphragm paralysis. (3) Bilateral diaphragm palsy has a unique presentation of orthopnea, dyspnea. Patient typically complaints of dyspnea in recumbent position similar to what our patient had also presented with. Pulmonary function test reveals restrictive abnormality, with reduced vital capacity and residual volume. Patients have profound sleep disturbance and loss of thoracic muscular tone during rapid eye movement sleep, which leads to nocturnal hypoxemia, hypercapnia, morning headaches and other symptoms of daytime somnolence. In the upright position, the weight of the abdominal contents tends to prevent excessive paradoxical motion and upward displacement of the diaphragm caused by the action of the intercostal and accessory muscles during maximal inspiration $[4,5]$. When these patients are in the supine position, this protective mechanism is lost, and patients have a marked reduction in vital capacity. Transdiaphragmatic measure measurements with balloon tipped esophageal and stomach catheters is accepted standard diagnostic tests for diaphragmatic palsy [6]. The prognosis of diaphragm palsy depends on the underlying process and bilateral diaphragm palsy should be suspected in cases of new onset dyspnea, more severe in supine position. Our patient presented with delayed onset of symptom's following trauma which could be a result of progressing cervical edema. Diagnosed clinically, radiologically and on basis of spirometry, Which could of extreme importance in resource limited settings. However, in some cases diaphragm paralysis might be permanent, nocturnal positive pressure ventilation is the mainstay of treatment similar to our case.

\section{References}

1. Dube BP, Dres M (2016) Diaphragm Dysfunction: Diagnostic approaches and management strategies. J Clin Med 5: 113. [Crossref]

2. Iverson LI, Mittal A, Dugan DJ, Samson PC (1976) Injuries to the phrenic nerve resulting in diaphragmatic paralysis with special reference to stretch trauma. The American journal of surgery 132: 263-269.

3. Schram DJ, Vosik W, Cantral D (2001) Diaphragmatic paralysis following cervical chiropractic manipulation. Chest 119: 638-639. [Crossref]

4. Martha E Billings, Moira L Aitken, Joshua O Benditt (2008) Bilateral diaphragm paralysis: A challenging diagnosis. Respiratory Care 53: 10. [Crossref]

5. Sandham JD, Shaw DT, Buenter CA (1977) Acute supine respiratory failure due to bilateral diaphragmatic paralysis. Chest 72: 96-98. [Crossref]

6. Kreitzer SM, Feldman NT, Saunders NA (1978) Bilateral diaphragmatic paralysis with hypercapnic respiratory failure. Am J Med 65: 89-95. [Crossref]

Copyright: (C2021 Joshi A. This is an open-access article distributed under the terms of the Creative Commons Attribution License, which permits unrestricted use, distribution, and reproduction in any medium, provided the original author and source are credited. 